Published in J. Low Temp. Phys. 139, 523-530 (2005).

\title{
Formation and Decay of Capillary Turbulence on the Charged Surface of Liquid Hydrogen
}

\author{
M.Yu. Brazhnikov, G.V. Kolmakov, A.A.Levchenko, \\ L.P. Mezhov-Deglin, and P.V.E. McClintock* \\ Institute of Solid State Physics RAS, 142432, Chernogolovka, Russia \\ * Department of Physics, Lancaster University, Lancaster, LA1 $4 Y B, U K$
}

We study the formation and the free decay of capillary turbulence on the charged surface of liquid hydrogen on the switching on, and off, of a narrowband driving force. It is observed that the decay begins from the high frequency spectral domain of the surface oscillations and is of a quasi-adiabatic character. The characteristic relaxation time of the whole turbulent cascade is close to the viscous damping time for capillary waves of frequency equal to the driving frequency.

$$
\text { PACS: } 47.27-i, 47.25+i
$$

\section{INTRODUCTION}

We report below the results of studies of the nonstationary processes involved in the formation and decay of capillary turbulence on the charged surface of liquid hydrogen. Some preliminary results of this work, including the main features of the decay of the turbulent state in a system of capillary waves, have already been published work $^{1,2}$.

It is known that the nonlinear interaction of capillary waves on the surface of liquid hydrogen is relatively strong, and that liquid hydrogen is a perfect fluid on which to undertake studies of the static and dynamical nonlinear phenomena occurring on liquid surfaces ${ }^{1}$. The possibility of driving the charged surface of liquid hydrogen directly through the application of electrical forces, provides an additional advantage.

It was shown in our earlier studies that a power-like Kolmogorov spectrum of capillary wave turbulence can be formed on the surface of liquid hydrogen over a very wide range of frequencies: from $100 \mathrm{~Hz}$ to $\approx 10 \mathrm{kHz}$,

$$
\left\langle\left|\eta_{\omega}\right|^{2}\right\rangle \sim \omega^{s}
$$




\section{M.Yu. Brazhnikov et al.}

The exponent is $s=-21 / 6$ in case of driving the surface by a low-frequency narrow-band force, and $s=-17 / 6$ in case of noisy driving, which is in accordance with expectations based on existing theory ${ }^{4}$. Here $\eta_{\omega}$ is the Fouriertransform over time of the surface elevation $\eta(r, t)$, and $\omega$ is the frequency the wave. At very high frequencies the power spectrum (1) is violated due to a change in the energy transfer mechanism from nonlinear wave transformation to viscous damping ${ }^{5}$.

Recently, we found ${ }^{3}$ that the character of the decay of capillary turbulence following removal of the driving force differs from what that might have been expected from the self-similar theory of nonstationary weak turbulent processes ${ }^{4}$, where the evolution of the spectrum is considered in the range of frequencies where viscous damping can be neglected. It was observed that a near steady-state power spectrum in the low frequency domain was stabilized after removal the driving force, whereas it is the high frequency part of the spectrum that is destroyed first. These observations agree well with our numerical solution ${ }^{2}$ of the kinetic equation for capillary waves.

In what follows we describe the results of new investigations of the decay and the formation of capillary turbulence on the charged surface of liquid hydrogen.

\section{EXPERIMENTAL RESULTS AND DISCUSSION}

\subsection{Experimental Setup}

The experimental arrangements were similar to those used in our earlier studies of steady state turbulence ${ }^{6}$. The measurements were made using an optical cell inside a helium cryostat. Hydrogen was condensed into a cup formed by a bottom capacitor plate and a guard ring $60 \mathrm{~mm}$ in diameter and $6 \mathrm{~mm}$ high. The layer of liquid was $6 \mathrm{~mm}$ thick. The top capacitor plate (a collector $60 \mathrm{~mm}$ in diameter) was located at the distance of $4 \mathrm{~mm}$ above the surface of the liquid. A two-dimensional positive charge layer was created just below the surface of the liquid with the aid of a radioactive plate placed at the bottom of the cup. The temperature of the liquid was $15.5 \mathrm{~K}$. Waves were excited on the charged surface by a periodic driving voltage applied between the guard ring and the upper electrode. They were detected from the variation of the total power $P(t)$ of a laser beam reflected from the oscillating surface, which was measured with a photodetector, sampled with an analogue-to-digital converter, and stored in a computer. Given the size of the light spot, the correlation function $I_{\omega}=\left\langle\left|\eta_{\omega}\right|^{2}\right\rangle$ of the surface elevation $\eta(\mathbf{r}, t)$ in frequency representation is directly proportional to the squared modulus of the Fourier transform of the detected signal, $I_{\omega}=\operatorname{const} P_{\omega}^{2}$ at frequencies above $50 \mathrm{~Hz}$. 
Formation and Decay of Capillary Turbulence

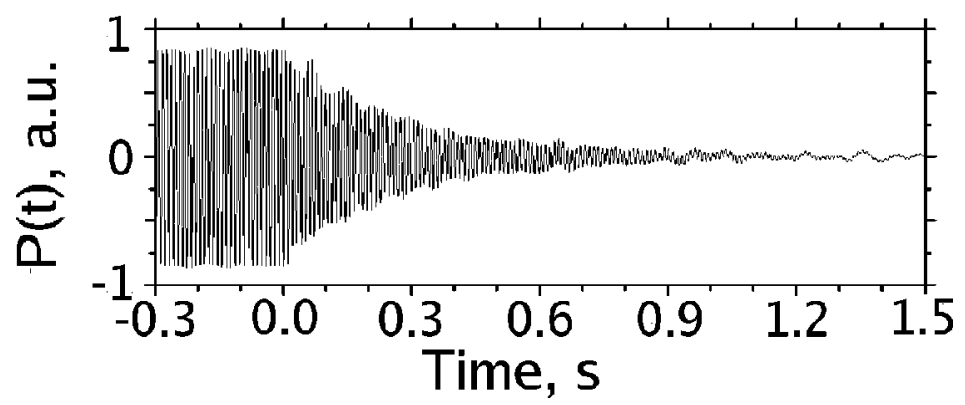

Fig. 1. Relaxation of oscillations of the charged liquid hydrogen surface after the cessation of external driving at frequency $\omega_{p} / 2 \pi=97 \mathrm{~Hz}$ at the time $t=0$.

In systems of finite size, the power spectrum of capillary turbulence is discrete; but, at frequencies much higher than the lowest resonant frequency ( $\approx 3 \mathrm{~Hz}$ in our experiments), the intrinsic spectrum of resonant frequencies becomes quasi-continuous due to viscous broadening of the resonances and/or nonlinear broadening for pumping rates exceeding a critical value. Under these conditions the distribution of turbulent oscillations of the surface of liquid in a cell of finite dimensions is close to that expected for the unrestricted surface (see theory ${ }^{7,8}$ and observations ${ }^{3,5,9,10}$ ).

\subsection{Free Decay of Capillary Turbulence}

The measurements were made on switching off the near-monochromatic pumping force applied at a frequency in the range from $30-400 \mathrm{~Hz}$. To establish the steady turbulent state at the surface of the liquid, a driving voltage was applied for $\approx 10 \mathrm{~s}$. After it was switched off, we observed relaxation oscillations of the surface. An example of such observation obtained on driving the surface at the frequency $\omega_{p} / 2 \pi=97 \mathrm{~Hz}$ is shown in Fig. 1 .

The instantaneous power spectrum $P_{\omega}^{2}$ of the nonstationary surface oscillations was calculated by application of the short-time Fourier transform ${ }^{11}$ to the measured signal $P(t)$.

Figure 2 shows the instantaneous spectra $P_{\omega}^{2}$ calculated for times (a) $t=$ $0.031 \mathrm{~s},(\mathrm{~b}) t=0.49 \mathrm{~s}$ and (c) $t=1.07 \mathrm{~s}$ after removal of the driving force of $\omega_{p} / 2 \pi=97 \mathrm{~Hz}$. At times close to $t=0$ the calculated spectrum of surface oscillations is close to the steady-state distribution of capillary waves observed in earlier experiments using a narrow-band driving force ${ }^{5}$; the initial (steady-state) boundary frequency of the inertial interval was $\omega_{b} / 2 \pi \approx 5 \mathrm{kHz}$. The main spectral peak in Fig. 2(a) is at the driving frequency of $97 \mathrm{~Hz}$, and the peaks at harmonics of the driving frequency form a cascade with a power 
M.Yu. Brazhnikov et al.
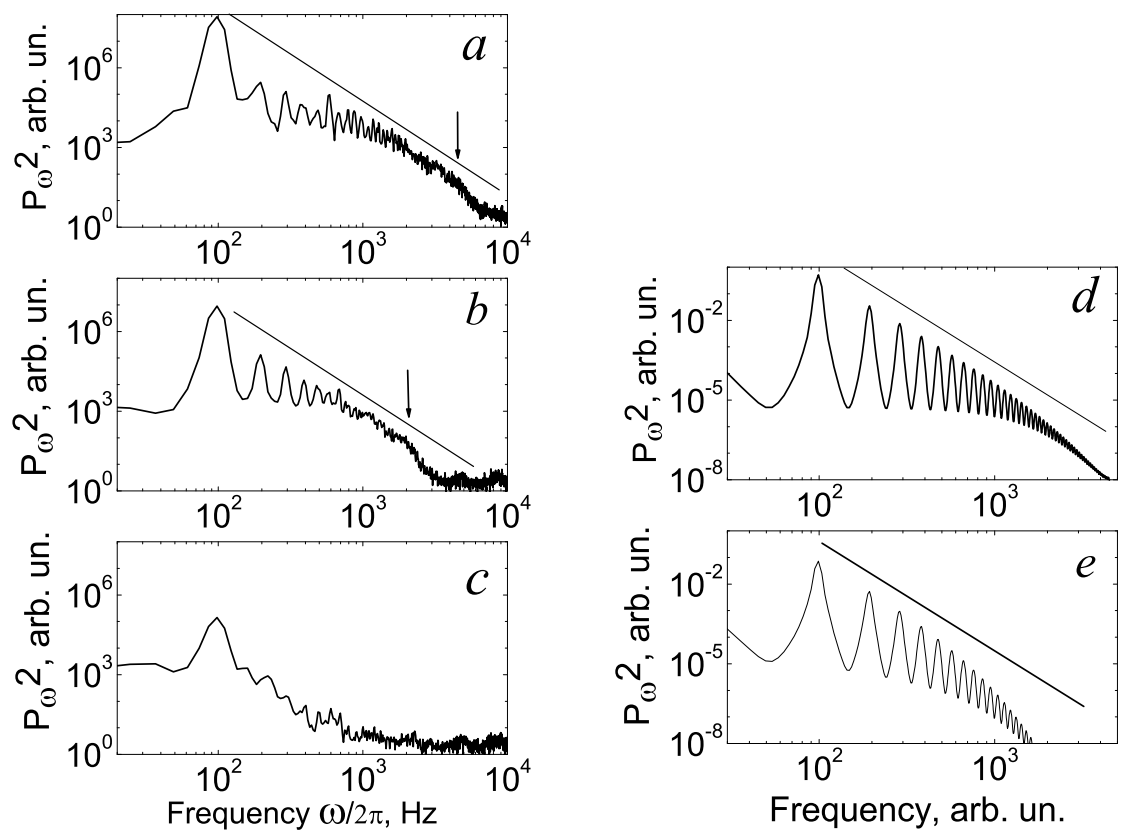

Fig. 2. Instantaneous spectra of surface oscillations calculated for different time intervals after removal of the driving force at frequency $\omega_{p} / 2 \pi=97 \mathrm{~Hz}$ : (a) $t=$ $0.031 \mathrm{~s}$, (b) $t=0.49 \mathrm{~s}$ and (c) $t=1.07 \mathrm{~s}$. The straight line corresponds to the powerlaw dependence $P_{\omega}^{2} \sim \omega^{-7 / 2}$. The arrows in (a) and (b) mark the positions of the high-frequency boundary of the inertial range. Plots (c) and (d) show the results of our numerical calculations of decay of the turbulent spectra after removal the narrow-band driving force, for times corresponding to the experimental observations shown in plots (a) and (b).

dependence $P_{\omega}^{2} \sim \omega^{-3.5}$ in the peak amplitudes, in agreement both with our previous observations ${ }^{5}$ and with theory ${ }^{4}$. The arrow marks the high frequency boundary of the inertial range. It is evident from Fig. 2(a) that the boundary frequency of the inertial interval has moved down from its initial value $\omega_{b} / 2 \pi \approx 5 \mathrm{kHz}$ at $t=0.031$ to $\omega_{b} / 2 \pi \approx 2 \mathrm{kHz}$ at $t=0.49 \mathrm{~s}$. Figure 2(c) shows the final stage in the decay of turbulence at $t=1.07 \mathrm{~s}$, when only few harmonics are excited.

Plots (c) and (d) show the results of our numerical calculations of decay of the turbulent spectra after removal the narrow-band driving force, for two moments of time corresponding to experimental observations shown in plots (a) and (b).

It is remarkable that the low-frequency part of the experimentally observed spectrum of surface oscillations can still be well-described by the same 


\section{Formation and Decay of Capillary Turbulence}

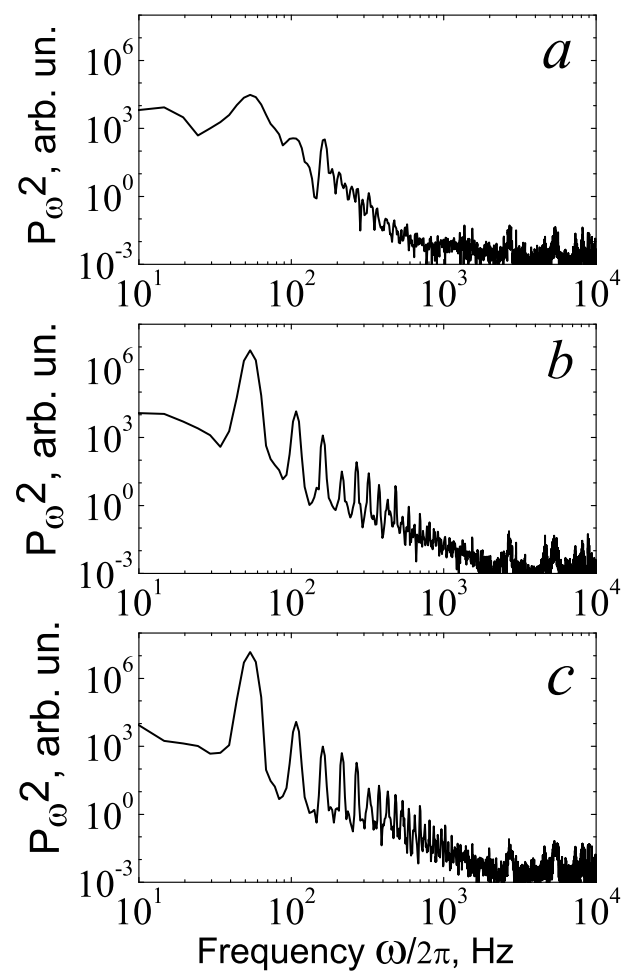

Fig. 3. Instantaneous spectra of surface oscillations calculated for different time intervals after switching on the driving force at the frequency $\omega_{p} / 2 \pi=56 \mathrm{~Hz}$ : (a) $t=$ $0 \mathrm{~s}$ (at moment of time immediately before the switching on the driving force), (b) $t=0.58 \mathrm{~s}$ and (c) $t=1.51 \mathrm{~s}$.

power law $P_{\omega}^{2} \sim \omega^{-3.5}$ as is valid for the steady-state turbulent distribution during a relatively long time interval $t<0.6 \mathrm{~s}$ after removal of the driving force. The decay of the turbulence starts from the high frequency domain of the spectrum and is accompanied by movement of the high frequency boundary $\omega_{b}$ of the inertial interval towards the low frequency domain. It can clearly be seen in Fig. 2(a,b,c) that the amplitude of the surface oscillations at the driving frequency exceeds noticeably the amplitudes of waves at the harmonics at all times during the decay. These observations differ significantly from what could be expected from the self-similar theory of nonstationary turbulent processes ${ }^{4}$, which was developed for cases where the dissipation is strictly equal to zero. Note that we do not observe any transition processes (for example, front propagation from low to high frequencies) after removal of the driving force. This means that the presence of finite 
M.Yu. Brazhnikov et al.

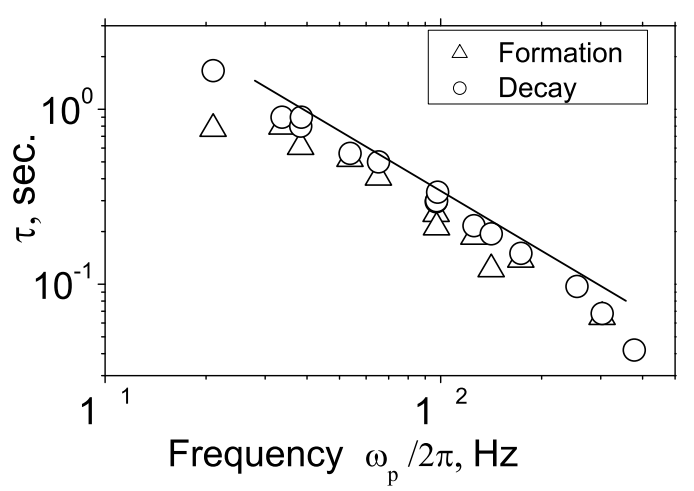

Fig. 4. Effective relaxation time $\tau$ of the surface oscillations plotted as a function of the driving frequency $\omega_{p}$ after removal of the driving force (open circles) and switching on the driving force (triangles). The solid line corresponds to the viscous damping time for a capillary wave $\tau_{v}$ with frequency $\omega=\omega_{p}$, calculated from known parameters of liquid hydrogen.

viscous damping of the waves causes a qualitative change in the character of relaxation of nonlinear surface waves. We may infer that the shape of the low frequency (power-like) part of the spectrum is stabilized by a fast redistribution of energy between waves within the inertial range of frequencies. This is why the observed spectrum remains close to quasiequilibrium over relatively long times following the start of the decay.

The results shown in Fig. 2(d,e) were obtained by numerical integration of the kinetic equation for capillary waves. In our preliminary work ${ }^{2}$ we used the model differential equation for the envelope of the spectrum. In the present computations, however, we have integrated numerically the whole kinetic equation for capillary waves. The results of the two computations look very similar and are in satisfactory agreement with the experimental observations.

We have shown that, at large times, the decrease in amplitude of low frequency waves occurs mainly on account of viscous damping; the nonlinear interaction with high frequency oscillations is negligibly small due to the smallness in amplitude of the high frequency waves. Waves of frequency much higher than that of the driving force are excited directly by interaction with low frequency waves. This means that under the conditions of real experiments (i.e. for finite width of the inertial range, limited by viscosity at frequencies of $5-10 \mathrm{kHz}$ ) the nonlocal interaction between capillary waves is comparable with this local interaction of waves. This accounts convincingly for the observed peculiarities in the decay of capillary turbulence on the 


\section{Formation and Decay of Capillary Turbulence}

surface of liquid hydrogen. In particular it explains why the dependence on the driving frequency of the effective relaxation time of surface oscillations is close to the dependence typical of linear waves, see below.

\subsection{Formation of Capillary Turbulence}

Figure 3 shows the instantaneous spectra $P_{\omega}^{2}$ calculated for times (a) $t=$ $0 \mathrm{~s}$, (b) $t=0.58 \mathrm{~s}$ and (c) $t=1.51 \mathrm{~s}$ after switching on the driving force at a frequency $\omega_{p} / 2 \pi=56 \mathrm{~Hz}$. Figure $3(\mathrm{a})$ shows the spectrum of surface oscillations, which was caused by low-level external noise excitations at moment of time immediately before the switching on the driving force. Fig. 3(b,c) demonstrate the formation of the turbulent spectrum at subsequent moments of time.

It can be seen from Figs. 2(a,b,c) and 3(a,b,c) that at each moment of time after switching off and on the driving force the spectra of surface oscillations are similar to the steady-state spectra, which were observed in our previous experiments at sufficiently large amplitudes. This means that both the processes of decay, and the processes of formation, of turbulence are controlled by the same relaxation mechanisms.

It was found earlier ${ }^{2,3}$ that the decreasing signal amplitude following removal of the driving force could be well-described by the exponential function $P(t) \sim \exp (-t / \tau)$, where $\tau$ is the effective relaxation time. The dependence of $\tau$ on frequency $\omega_{p} / 2 \pi$ is shown by open circles in Fig. 4 . The straight line shows the dependence of the viscous (linear) damping time for capillary waves $\tau_{v}=\nu(\rho / \alpha)^{2 / 3} \omega^{4 / 3}$ at frequency $\omega=\omega_{p}$, calculated from the known parameters $^{12}$ of liquid hydrogen at $15.5 \mathrm{~K}$ (here $\nu$ is the viscosity, $\rho$ is the density and $\alpha$ is the surface tension of liquid hydrogen). It is clearly evident from Fig. 4 that the measured relaxation time $\tau$ for the decay of the turbulent cascade is close to the viscous damping time $\tau_{v}$ of the waves. The triangles in Fig. 4 shows the characteristic time of formation of turbulent cascade after switching on the driving force at the frequency range from 20 to $300 \mathrm{~Hz}$. It is evident from Fig. 4 that the time of formation is close to the time of decay and to the time of viscous attenuation of the surface waves at the frequency $\omega_{p}$.

\section{CONCLUSIONS}

The experimental data and the results of our numerical computations presented above and in earlier papers ${ }^{2,3}$ have shown that, after removal of a low frequency driving force, we observe a quasi-adiabatic decay of the turbulent cascade of capillary waves on the surface of liquid hydrogen. The 


\section{M.Yu. Brazhnikov et al.}

decay starts from the high frequency domain of the spectrum, and the effective relaxation time of the cascade is close to the viscous damping time for waves of frequency equal to the driving frequency. The energy-containing range stays in the low frequency domain throughout the decay. This indicates that viscous losses at all frequencies are of central importance for the correct interpretation of decay processes in a system of nonlinearly interacting capillary waves. Observations of the formation of the cascade are in satisfactory agreement with those conclusions.

\section{ACKNOWLEDGEMENTS}

The authors are very grateful to V.E.Zakharov, E.A. Kuznetsov, M.T.Levinsen, and A.N. Silchenko for many useful discussions, and to V.N. Khlopinskii for assistance in preparation of the experiments. The investigations are supported in part by INTAS (grant 2001-0618), by RFBR (grant 03-02-16865) and by the Ministry of Education and Science of RF (program "Quantum phenomena at low and ultralow temperatures"). G.V.K. acknowledges also support from the Science Support Foundation (Russia).

\section{REFERENCES}

1. M.Y. Bazhnikov, G.V. Kolmakov, A.A. Levchenko, L.P. Mezhov-Deglin, Low Temp. Phys. $\mathbf{2 7}_{(9-10)}, 876$ (2001).

2. G.V. Kolmakov, A.A. Levchenko, M.Yu. Brazhnikov, L.P. Mezhov-Deglin, A.N. Silchenko, and P.V.E. McClintock, Phys. Rev. Lett. 93, 074501 (2004).

3. M.Yu. Brazhnikov, G.V. Kolmakov, A.A. Levchenko, L.P. Mezhov-Deglin, A.N. Silchenko, and P.V.E. McClintock, JETP Lett. 80 $(2), 90$ (2004).

4. V.Zakharov, V. L'vov, and G. Falkovich, Kolmogorov Spectra of Turbulence, Vol. 1 (Springer, Berlin 1992).

5. M. Brazhnikov, A. Levchenko, and G. Kolmakov, JETP 95, 447 (2002).

6. M.Yu. Brazhnikov, A.A. Levchenko, and L.P. Mezhov-Deglin, Instr. Exp. Techn. 45, 758 (2002).

7. A.N. Pushkarev and V.E. Zakharov, Phys. Rev. Lett. 76, 3320 (1996).

8. A.I. Dyachenko, A.O. Korotkevich, and V.E. Zakharov, JETP Lett. 77, 477 (2003).

9. W. Wright, R. Budakian, and S. Putterman, Phys. Rev. Lett. 76, 4528 (1996).

10. E. Henry, P. Alstrom, and M.T. Levinsen, Europhys. Lett. 52, 27 (2000).

11. S. Mallat, A Wavelet Tour of Signal Processing (Academic Press, New York 1997); R. Gribonval and E. Bacry, IEEE Trans.- Signal Processing 51, 101 (2003).

12. B.I. Verkin (Editor), Properties of Condensed Phases of Hydrogen and Oxygen (Kiev, Naukova Dumka 1984). 